\title{
PERCEPCIÓN DE LAS FORTALEZAS DE LOS ADOLESCENTES DURANTE EL CONFINAMIENTO POR COVID-19
}

\author{
Erika Yadira Macías Mozqueda, Norma A. Ruvalcaba Romero, \\ Mercedes Gabriela Orozco Solís y Hector Ruben Bravo-Andrade \\ Universidad de Guadalajara \\ México
}

\begin{abstract}
RESUMEN
El confinamiento por Covid-19 nos llevó a comparar las fortalezas externas e internas de los adolescentes antes y durante el confinamiento. Con un estudio no experimental ex post facto en una muestra de 130 estudiantes de secundaria, con una edad promedio de 13 años, $51 \%$ hombres y $49 \%$ mujeres, se les aplicó la escala de Fortalezas. Los resultados indican que se perciben algunos cambios en el confinamiento diferenciados por el sexo. Entre ellos, un incremento significativo en el apoyo de la madre, $\mathrm{t}(65)=$ $-2.83, \mathrm{p}=0.006, \mathrm{~d}=0.071$ para hombres, y $\mathrm{t}(62)=-3.15, \mathrm{p}=0.003, \mathrm{~d}=0.057$ para mujeres; disminución en la supervisión de la madre, $\mathrm{t}(65)=2.70, \mathrm{p}=0.009, \mathrm{~d}=0.137$ en hombres y $\mathrm{t}(62)=2.81, \mathrm{p}=0.007, \mathrm{~d}=0.093$ en mujeres, así como otras fortalezas que se detallan en el documento.
\end{abstract}

Palabras Clave:

adolescentes, familia, fortalezas, confinamiento y Covid-19

\section{Perception of the strengths of adolescents DURING CONFINEMENT BY COVID-19}

\begin{abstract}
The Covid-19 confinement led us to compare the external and internal strengths of adolescents before and during lockdown. With a non-experimental ex post facto study in a sample of 130 secundary students, with an average age of 13 years, $51 \%$ men and $49 \%$ women, the Strengths scale was applied. The results indicate that they did perceive some changes in confinement differentiated by sex. Among them, a significant increase in the support of the mother, $t(65)=-2.83, p=0.006, d=0.071$ for men and $t(62)=-3.15, p=0.003, d=0.057$ for women; decrease in mother's supervision, $t(65)=2.70, p=0.009, d=0.137$ in men and $\mathrm{t}(62)=2.81, \mathrm{p}=0.007, \mathrm{~d}=0.093$ in women as well as other strengths that are detailed in the document.
\end{abstract}

\section{Keywords:}

teenagers, family, strengths, lockdown, Covid-19

Bitácora del Artículo:

| Recibido: 21 de Abril de 2021 | Aceptado: 4 de Junio de 2021 | Publicado en línea: Julio-Diciembre de 2021 | 
Artículo Empírico | Percepción de las fortalezas de los adolescentes... I Macías-Mozqueda, Ruvalcaba-Romero, Orozco-Solís, y Bravo-Andrade

\title{
Autoría y Derechos de Propiedad Intelectual
}

\section{PERCEPCIÓN DE LAS FORTALEZAS DE LOS ADOLESCENTES DURANTE EL CONFINAMIENTO POR COVID-19}

\author{
Erika Yadira Macías Mozqueda, Norma A. Ruvalcaba Romero, \\ Mercedes Gabriela Orozco Solís y Hector Ruben Bravo-Andrade \\ Universidad de Guadalajara \\ México
}

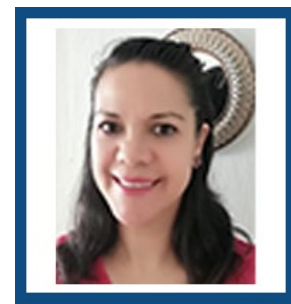

Erika Yadira Macías Mozqueda Universidad de Guadalajara

Correo: erika.macias@academicos.udg. $\mathrm{mx}$

Doctorado en Gestión de la Educación Superior por la Universidad de Guadalajara. Profesora en la Licenciatura en Psicología y en la Maestría en Psicología Educativa del CUCS en UdeG. Las líneas de investigación en las que trabaja son Psicología y Bienestar así como Educación Superior y Trayectorias Laborales. Miembro del Sistema Nacional de Investigadores Nivel Candidato.

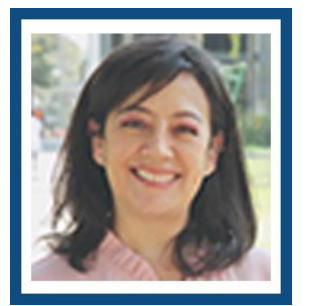

Norma A. Ruvalcaba Romero Universidad de Guadalajara Correo:norma.ruvalcaba@academicos. udg.mx

Doctorado en Inteligencia Emocional en la Universidad de Málaga. Es Miembro del Sistema Nacional de Investigadores. Docente de la carrera de Psicología en el Centro Universitario de Ciencias de la Salud, de los posgrados de Psicología en el Centro Universitario del Sur y de la Maestría en Innovación Social y Gestión del Bienestar en el Centro Universitario de Ciencias Económico-Administrativas.

\section{CONTRIBUCIÓN DE LOS AUTORES}

Erika Yadira Macias Mozqueda diseñó el estudio y participó en todos los rubros del artículo. | Norma A. Ruvalcaba Romero participó en la elaboración de la metodología, análisis y resultados. | Mercedes Gabriela Orozco Solís participó en la discusión y conclusiones. | Hector Rubén Bravo-Andrade participó en los resultados y conclusiones | Los cuatro autores participaron en la revisión y corrección del manuscrito.

\section{AGRADECIMIENTOS}

A la institución educativa que brindó las facilidades para el levantamiento de datos.

\section{DATOS DE FILIACIÓN DE LOS AUtORES}

Centro Universitario de Ciencias de la Salud de la Universidad de Guadalajara.

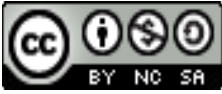

Copyright: (c) 2021 Macías-Mozqueda, E. Y.; Ruvalcaba-Romero, N.A.; Orozco-Solís, M. G.; y Bravo-Andrade, H, R.

Este es un artículo de acceso abierto distribuido bajo los términos de la licencia Creative Commons Reconocimiento-NoComercial 4.0 Internacional, por lo que su contenido gráfico y escrito se puede compartir, copiar y redistribuir total o parcialmente sin necesidad de permiso expreso de sus autoras con la única condición de que no se puede usar con fines directamente comerciales y los términos legales de cualquier trabajo derivado deben ser los mismos que se expresan en la presente declaración. La única condición es que se cite la fuente con referencia a la Revista Digital Internacional de Psicología y Ciencia Social y a sus autores. 


\section{TABLA DE CONTENIDO}

INTRODUCCIÓN

EL CONFINAMIENTO

LAS FAMILIAS EN CONFINAMIENTO

MÉTODO

Participantes, 209

Materiales, 209

PROCEDIMIENTO

Mediciones, 209

Análisis estadísticos, 209

REsultados

Discusıón

CONCLUSIONES 


\section{INTRODUCCIÓN}

$\mathbf{E}$ I enfoque del Desarrollo Positivo de los Jóvenes (PYD, por sus siglas en inglés) estudia los factores que favorecen el desarrollo integral del adolescente partiendo del potencial de cambio como una fortaleza fundamental en la que se puede construir, y por tanto influir, en una dirección positiva, por lo que se enfocan en los activos del desarrollo mediante programas para fomentar el PYD (Lerner y Lerner, 2011).

El modelo de fortaleza desarrollado por el Search Institute of Minnesota, que contribuye a la evaluación de PYD, considera que los jóvenes tienen fortalezas internas y externas que contribuyen en su desarrollo positivo. Se agrupan en externas las que se producen en la interacción entre adulto-adolescente-pares y se refuerzan en las instituciones, y las internas, que se desarrollan de manera gradual a partir de las experiencias de vida (Betancourt-Ocampo, Andrade-Palos y Marín-Martínez, 2018).

Aunque se ha informado que hay diferencias en cuanto a las fortalezas psicológicas en adolecentes según el sexo (Brown, Blanchard y McGrath, 2020; Reyes y Farragut, 2016), de manera general las y los adolescentes con mayores fortalezas personales y sociales tienen más posibilidades de lograr bienestar y éxito, y menor posibilidad de involucrarse en situaciones de riesgo (Benson, Scales, Hamilton y Sesma, 2006).

Estudios empíricos evidencian que las fortalezas en adolescentes los protegen de consumir tabaco (Sánchez-Xicotencatl y Andrade-Palos, 2019), consumir sustancias ilegales (Andrade-Palos, Betancourt-Ocampo, Moreno-Carmona y Alvis-Rizo, 2017) e intentos de suicidio (Moreno-Carmona, Andrade-Palos y Betancourt-Ocampo, 2018). Asimismo se les ha asociado de manera negativa con indicadores de afecto negativo como depresión, ansiedad y estrés (Ovejero, Cardenal y Ortiz-Tallo, 2016).

Por otra parte, las fortalezas predicen de manera significativa las estrategias de afrontamiento y bienestar psicológico en adolescentes (García-Álvarez, Hernandez-Lalinde, Cobo-Rendón, Espinosa-Castro y Soler, 2020), así como la felicidad y la salud mental en este grupo poblacional (Reyes y Ferragut, 2016). Asimismo, se relacionan de modo positivo con la satisfacción vital, la resiliencia y la salud (Ovejero et al., 2016).
Respecto a las fortalezas externas, el tipo de apoyo y comunicación que percibe el adolescente de sus padres se ha asociado al desarrollo de sintomatología depresiva (Andrade-Palos, Betancourt-Ocampo, Vallejo-Casarín, Segura-Celis y Rojas-Rivera 2012; Ruvalcaba-Romero, Gallegos-Guajardo, Caballo y Villegas-Guinea, 2016). Por otro lado, la aceptación emocional de los padres fomenta un desarrollo adecuado, mayor iniciativa social (Florenzano et al., 2009) y mayor experimentación de emociones positivas (Ruvalcaba-Romero, Orozco-Solís, Gallegos-Guajardo y Nava-Fuerte, 2018).

\section{El confinamiento}

En 2020 el confinamiento que se estableció para reducir la pandemia de Covid-19 redujo las actividades sociales de la población en general, los adolescentes dejaron de asistir a la escuela, lo que se sumó a la reducción de la interacción presencial con sus pares. Dicha dinámica representa una adversidad psicosocial que pone en riesgo la estabilidad familiar (Palacio-Ortiz, Londoño-Herrera, Nanclares-Márquez, Robledo-Rengifo y Quintero-Cadavid, 2020), por lo que la relación con sus progenitores adquiere mayor importancia al incrementarse el tiempo en casa.

Los efectos de la pandemia por Covid-19 en adolescentes se han estudiado desde diversas perspectivas; sin embargo, una dificultad en el abordaje ha sido que la salud mental de la población adolescente podría verse afectada por el confinamiento, aunque al ser una etapa de cambios no todas las reacciones pueden calificarse como patológicas (Galiano-Ramírez, Prado-Rodríguez y Mustelier-Becquer, 2020).

Al comparar los efectos en el estado de ánimo, la empatía y la conducta prosocial en un grupo de adolescentes holandeses que participaban en un estudio previo a la pandemia contra los efectos de los primeros meses de confinamiento, encontraron que los niveles de preocupación empática y las oportunidades para acciones prosociales disminuyeron, pero mostraron resiliencia, aumento de los niveles de toma de perspectiva y vigor, disminución de los niveles de tensión y altos niveles de donación para contribuir de manera significativa a la sociedad (Van de Groep, Zanolie, Green, Sweijen y Crone, 2020)

En un grupo de padres y adolescentes holandeses relativamente sanos entre quienes se comparó la primera etapa de la pandemia con un periodo de referencia previo, no se encontraron afectos negativos (Janssen et al., 2020). Un estudio hecho en Finlandia acerca de las estrategias de afrontamiento que utilizaron las familias en la pandemia demostró que desarrollaron muchos medios para sobrellevar el confinamiento, lo que indica la función fundamental 
de las relaciones conyugales y parentales para hacer frente al problema (Salin, Kaittila, Hakovirta y Antilla, 2020).

Respecto a las afectaciones en los hábitos de alimentación y actividades físicas de los adolescentes como consecuencia del confinamiento por Covid-19, un estudio realizado en Italia, España, Chile, Colombia y Brasil reveló que se incrementó el consumo de alimentos fritos y dulces, así como patrones irregulares de alimentación y refrigerios frecuentes, lo que incrementa el riesgo de obesidad (Ruiz-Roso et al., 2020). También se redujo de modo significativo la participación en actividades físicas y la resistencia física de los adolescentes como consecuencia de las medidas de distanciamiento social aplicadas al sur de Croacia, sobre todo porque las actividades que hacían eran deportes organizados en establecimientos especializados (Sekulic, Blazevic, Gilic, Kvesic y Zenic, 2020).

Las actividades físicas de un grupo de adolescentes españoles se estudiaron en correlación con el autoconcepto durante el confinamiento por Covid-19; se encontró una asociación positiva entre las dimensiones del autoconcepto y el nivel de la actividad física, así como una asociación negativa entre el autoconcepto y el comportamiento sedentario. La familia y los compañeros son factores fundamentales para el desarrollo apropiado del autoconcepto emocional antes y durante el confinamiento (González-Valerio, et al., 2020). También se ha informado que durante el confinamiento ha habido un incremento de hábitos poco saludables, identificando un aumento en los problemas de alimentación y sueño, y una disminución de las actividades deportivas (Brooks et al., 2020; Paricio y Pando, 2020; Sandín, Valiente, García y Chorot, 2020).

Por otro parte, para adolescentes víctimas de bullying el confinamiento también significó la disminución de su ansiedad, depresión y ciberbullying, pero en el caso de los adolescentes que no han sido víctimas se reportó disminución del rendimiento académico (Gómez-León, 2021).

\section{LAS FAMILIAS EN CONFINAMIENTO}

Las dinámicas familiares tienen una función protagónica en las repercusiones de la pandemia en los adolescentes; el no tener cubiertas las necesidades básicas y la carencia de un entorno familiar y educativo protector impacta de modo directo en la salud mental (Galiano-Ramírez et al., 2020).

Las repercusiones del confinamiento en familias que ya estaban expuestas a la violencia intrafamiliar, sumadas a las dificultades económicas, incrementan las posibilidades de abusos, por lo que se exacerban los sín- tomas de niños y jóvenes con trastorno psiquiátrico previo (Palacio-Ortiz et al., 2020). Asimismo, la sintomatología depresiva se incrementó en los chicos conforme aumenta la edad, el tiempo del confinamiento y el conflicto familiar (Martínez-Pérez, Fernández-Fernández, Alcántara-López, López-Soler y Castro-Sáez, 2020).

El reto de la escuela en casa se ha superado cuando en las familias se tiene un nivel educativo superior o se hay estrategias de comunicación con los hijos y con los actores educativos; esto no es así en las familias con poca capacidad y tiempo para apoyar el proceso desde casa, lo que se suma a la brecha educativa (Nivela-Cornejo, Molina-Villacís y Campos-Vera, 2020).

Como efecto positivo del confinamiento y del incremento del trabajo a distancia, la mayoría de niños y adolescentes han percibido como positivo pasar más tiempo con sus padres (Serrano-Martínez, 2020), sumado a una mejoría de la relación de los adolescentes con sus padres, en particular con la madre (Cros, Preve, Revilla y Maristany, 2021), así como un incremento en la percepción de las madres acerca de las dificultades de sus hijos, lo que posibilitó el ajuste en los menores y mejoría en sus acuerdos (Martínez-Pérez et al., 2020).

Por otra parte, la calidad de la relación se ha afectado por la posibilidad de los padres para establecer cuidados, afecto y un entorno seguro para los adolescentes; sumado a esto, la mayoría de estas tareas se ha cubierto por la madre, lo que favorece la sobrecarga y dificulta mantener la supervisión (Galiano-Ramírez et al., 2020). Entre los factores que dificultan que los padres puedan mantener una supervisión adecuada se encuentran el incremento del trabajo a distancia, tener que salir a trabajar, y la educación desde casa (Nivela-Cornejo et al., 2020).

Con base en la información descrita, en los primeros meses del confinamiento las afectaciones a los adolescentes reportadas fueron en las actividades físicas en las que era necesario salir de sus casas, porque a pesar de que las familias modificaron sus actividades y rutinas, encontraron nuevas maneras de adaptación. Los hábitos de alimentación se modificaron en perjuicio de la población adolescente. Sin embargo, en las familias con antecedentes, la violencia y las dificultades económicas potencializaron las dificultades, afectando la salud mental de los adolescentes. Por tanto, al modificarse la interacción de los adolescentes con los adultos, con los otros adolescentes y con sus pares, y al ser esta interacción el espacio donde se desarrollan las fortalezas, planteamos la siguiente hipótesis: Al cambiar la manera de interacción de los adolescentes con los otros como consecuencia del confinamiento, también se modifica la percepción de las fortalezas externas e internas. 
El objetivo de este estudio es comparar la percepción de los adolescentes de sus fortalezas internas y externas antes y durante los primeros meses del confinamiento. La pregunta de investigación es: ¿Los adolescentes perciben cambios en sus fortalezas externas e internas antes y durante el confinamiento?

\section{Método}

\section{Participantes}

La muestra es no probabilística por conveniencia; estuvo constituida por 130 adolescentes que cursaban estudios de los tres grados de secundaria en una escuela pública de la ciudad de Guadalajara. El 51\% son hombres y $49 \%$ mujeres, con rango de edad de 12 a 16 años $(X=$ 13.01 , D.T. $=0.87)$. Esta muestra no es representativa de los adolescentes mexicanos, por lo que los resultados no son generalizables.

\section{Materiales}

Se aplicaron subescalas de la escala de fortalezas (Betancourt-Ocampo, Andrade-Palos, González y Sánchez-Xicotencatl, 2018). Se trata de un instrumento con una estructura tipo Likert de cinco puntos que evalúa recursos internos (responsabilidad, toma de decisiones, resistencia ante la presión de pares, evitación de conductas de riesgo y percepción de la importancia de la salud y la religión) y recursos externos (supervisión y apoyo de padre y madre, presencia de amigos con hábitos saludables, presencia de amigos sin conductas de riesgo y reglas al interior del hogar).

En su versión original, los autores informaron los siguientes índices de alfa de Cronbah para las dimensiones de los recursos internos: responsabilidad $(\mathrm{a}=$ $0.714)$, toma de decisiones $(a=0.859)$, resistencia ante la presión de pares $(\mathrm{a}=0.786)$, evitación de conductas de riesgo $(\mathrm{a}=0.800)$, percepción de la importancia de la salud $(\mathrm{a}=0.881)$ y percepción de la religión $(\mathrm{a}=0.927)$; por otro lado se reportan los siguientes índices para los recursos externos: apoyo y supervisión del padre $(\mathrm{a}=$ $0.9263)$, apoyo de la madre $(\mathrm{a}=0.932)$, presencia de amigos con hábitos saludables $(\mathrm{a}=0.527)$ y presencia de amigos sin conductas de riesgo $(\mathrm{a}=0.729)$.

\section{Procedimiento}

Cuando el proyecto fue autorizado por la dirección del plantel escolar se procedió al envío de la carta de consentimiento informado para ser revisado y firmado por los padres, madres o tutores de los adolescentes; en dicho documento se hace constar que la participación es voluntaria, confidencial y sin riesgo para los informantes. Los adolescentes también firmaron un asentimiento informado en el que se hace de su conocimiento que su participación es voluntaria, confidencial, y se pueden retirar en cualquier momento y sin perjuicio alguno. La escala se aplicó de manera electrónica (por formulario Google) sólo a los estudiantes cuyos padres habían firmado el consentimiento informado; los datos se recabaron en octubre de 2020 después de siete meses de confinamiento.

\section{Mediciones}

En este estudio se aplicaron las siguientes subescalas (se indica entre paréntesis el alfa de Cronbach obtenido en esta muestra): apoyo de la madre $(\mathrm{a}=0.943)$, supervisión de la madre $(a=0.866)$, apoyo y supervisión del padre $(a=0.972)$, amigos saludables $(a=0.643)$, amigos sin conductas de riesgo $(a=0.764)$, reglas en el hogar $(\mathrm{a}=0.776)$, toma de decisiones $(\mathrm{a}=0.860)$, responsabilidad $(\mathrm{a}=0.722)$ y percepción de la importancia de la Salud $(\mathrm{a}=0.881)$.

Es necesario indicar que para los objetivos de este estudio - para contrastar las respuestas- se aplicó la escala preguntando acerca de la percepción de dichas fortalezas antes y durante el confinamiento.

\section{Análisis estadísticos}

Se hicieron análisis descriptivos y la prueba t para muestras relacionadas con el objetivo de lograr una comparación de media. Los datos se analizaron con el sistema estadístico SPSS v.25.

\section{Resultados}

Con el objetivo de comparar la percepción de las fortalezas internas y externas antes y durante el confinamiento de las y los adolescentes, se hizo una prueba para muestras relacionadas diferenciando por sexo. Los resultados se muestran en las tablas 1 y 2 , donde además se incluyen los estadísticos descriptivos.

Los datos de las tablas indican que los cambios se identifican sólo en algunas variables. En la que se percibió incremento significativo para ambos sexos fue en la correspondiente al apoyo de la madre, en contraparte a la variable de supervisión de la madre - cuyo cambio fue hacia el decremento-. Por otro lado, en el caso de las mujeres disminuyó la percepción de los hábitos saludables de los amigos (que en específico se refieren a llevar una dieta saludable, practicar deportes, dormir suficiente y utilizar cinturón de seguridad). En el caso de los varones 
se percibió disminución en la subescala de responsabilidad (que implica ser puntual en sus clases y cumplir con los compromisos), así como un aumento en las habilidades para la toma de decisiones (en las que se incluyen la capacidad para buscar información, identificar soluciones a los problemas, así como sus consecuencias).

\section{Tabla 1.}

Diferencias en la percepción de las fortalezas internas y externas antes y durante el confinamiento por Covid 19, en hombres.

\begin{tabular}{|c|c|c|c|c|c|c|c|}
\hline \multirow{2}{*}{ VARIABLES } & \multicolumn{2}{|c|}{$\begin{array}{l}\text { ANTES DEL } \\
\text { CONFINAMIENTO }\end{array}$} & \multicolumn{2}{|c|}{$\begin{array}{l}\text { DURANTE EL } \\
\text { CONFINAMIENTO }\end{array}$} & \multirow[b]{2}{*}{ T(GL) } & \multirow[b]{2}{*}{$\mathbf{P}$} & \\
\hline & MEDIA & DE & Media & DE & & & D \\
\hline
\end{tabular}

Fortalezas
externas

\begin{tabular}{|c|c|c|c|c|c|c|c|}
\hline $\begin{array}{l}\text { Apoyo de la } \\
\text { madre }\end{array}$ & 3.67 & 0.90 & 3.76 & 0.89 & $\begin{array}{l}-2.83 \\
(65)\end{array}$ & 0.006 & 0.071 \\
\hline $\begin{array}{l}\text { Supervisión } \\
\text { de la madre }\end{array}$ & 4.16 & 0.91 & 3.99 & 0.84 & $\begin{array}{l}2.70 \\
(65)\end{array}$ & 0.009 & 0.137 \\
\hline $\begin{array}{l}\text { Apoyo y } \\
\text { supervisión } \\
\text { del padre }\end{array}$ & 3.22 & 1.32 & 3.24 & 1.29 & $\begin{array}{l}-0.57 \\
(65)\end{array}$ & 0.571 & - \\
\hline $\begin{array}{l}\text { Amigos } \\
\text { saludables }\end{array}$ & 3.20 & 0.87 & 3.15 & 0.90 & $\begin{array}{l}1.44 \\
(65)\end{array}$ & 0.153 & - \\
\hline $\begin{array}{l}\text { Amigos sin } \\
\text { conductas } \\
\text { de riesgo }\end{array}$ & 4.70 & 0.52 & 4.73 & 0.46 & $\begin{array}{l}-1.22 \\
(65)\end{array}$ & 0.223 & - \\
\hline $\begin{array}{l}\text { Reglas en el } \\
\text { hogar }\end{array}$ & 3.96 & 0.86 & 3.93 & 0.85 & $\begin{array}{l}0.56 \\
(65)\end{array}$ & .578 & - \\
\hline \multicolumn{8}{|l|}{$\begin{array}{l}\text { Fortalezas } \\
\text { internas }\end{array}$} \\
\hline $\begin{array}{l}\text { Responsa- } \\
\text { bilidad }\end{array}$ & 3.90 & 0.75 & 3.83 & 0.74 & $\begin{array}{l}2.49 \\
(65)\end{array}$ & 0.015 & 0.066 \\
\hline $\begin{array}{l}\text { Toma de } \\
\text { decisiones }\end{array}$ & 3.81 & 0.88 & 3.88 & 0.86 & $\begin{array}{l}-2.41 \\
(65)\end{array}$ & 0.019 & 0.057 \\
\hline $\begin{array}{l}\text { Importancia } \\
\text { de la salud }\end{array}$ & 3.92 & 1.21 & 3.98 & 1.18 & $\begin{array}{l}-1.07 \\
(65)\end{array}$ & 0.288 & - \\
\hline
\end{tabular}

\section{DisCusión}

El objetivo de este trabajo es comparar la percepción que tienen los adolescentes de sus fortalezas internas y externas antes y durante los primeros meses del confinamiento. Si bien los resultados demuestran que los adolescentes perciben algunas diferencias, sobre todo en las fortalezas externas, los tamaños del efecto de dichos cambios fueron muy bajos. No obstante, la tendencia de los datos coincide con los siguientes autores.

La fortaleza externa con más significativos fue el apoyo de la madre; se identificó un aumento durante los meses iniciales del periodo de confinamiento. Este resultado coincide con estudios previos que informan que pasar más tiempo con los padres derivado del con-

\section{Tabla 2.}

Diferencias en la percepción de las fortalezas internas y externas antes y durante el confinamiento por Covid 19, en mujeres.

\begin{tabular}{|c|c|c|c|c|c|c|c|}
\hline \multirow{2}{*}{ VARIABLES } & \multicolumn{2}{|c|}{$\begin{array}{l}\text { ANTES DEL } \\
\text { CONFINAMIENTO }\end{array}$} & \multicolumn{2}{|c|}{$\begin{array}{l}\text { DURANTE EL } \\
\text { CONFINAMIENTO }\end{array}$} & \multirow[b]{2}{*}{$T(G L)$} & \multirow[b]{2}{*}{$\mathbf{P}$} & \multirow[b]{2}{*}{ D } \\
\hline & Media & DE & MediA & DE & & & \\
\hline
\end{tabular}

Fortalezas

externas

\begin{tabular}{|c|c|c|c|c|c|c|c|}
\hline $\begin{array}{l}\text { Apoyo de la } \\
\text { madre }\end{array}$ & 3.86 & 1.15 & 3.95 & 1.08 & $\begin{array}{l}-3.15 \\
(62)\end{array}$ & 0.003 & .057 \\
\hline $\begin{array}{l}\text { Supervisión } \\
\text { de la madre }\end{array}$ & 4.24 & 0.91 & 4.12 & 0.92 & $\begin{array}{l}2.81 \\
(62)\end{array}$ & 0.007 & .093 \\
\hline $\begin{array}{l}\text { Apoyo y } \\
\text { supervisión } \\
\text { del padre }\end{array}$ & 3.09 & 1.44 & 3.13 & 1.42 & $\begin{array}{l}-1.27 \\
(62)\end{array}$ & 0.207 & - \\
\hline $\begin{array}{l}\text { Amigos } \\
\text { saludables }\end{array}$ & 3.21 & 0.77 & 3.04 & 0.77 & $\begin{array}{l}3.56 \\
(62)\end{array}$ & 0.001 & .156 \\
\hline $\begin{array}{l}\text { Amigos sin } \\
\text { conductas } \\
\text { de riesgo }\end{array}$ & 4.69 & 0.54 & 4.68 & 0.62 & $\begin{array}{l}0.15 \\
(62)\end{array}$ & 0.883 & - \\
\hline $\begin{array}{l}\text { Reglas en el } \\
\text { hogar }\end{array}$ & 3.96 & 0.92 & 3.88 & 0.95 & $\begin{array}{l}1.73 \\
(62)\end{array}$ & 0.088 & - \\
\hline \multicolumn{8}{|l|}{$\begin{array}{l}\text { Fortalezas } \\
\text { internas }\end{array}$} \\
\hline $\begin{array}{l}\text { Responsa- } \\
\text { bilidad }\end{array}$ & 4.07 & 0.86 & 4.03 & 0.86 & $\begin{array}{l}1.07 \\
(62)\end{array}$ & 0.289 & - \\
\hline $\begin{array}{l}\text { Toma de } \\
\text { decisiones }\end{array}$ & 3.92 & 0.90 & 3.89 & 0.89 & $\begin{array}{l}1.03 \\
(62)\end{array}$ & 0.302 & - \\
\hline $\begin{array}{l}\text { Importancia } \\
\text { de la salud }\end{array}$ & 4.21 & 1.15 & 4.29 & 1.13 & $\begin{array}{l}-1.30 \\
(62)\end{array}$ & 0.199 & - \\
\hline
\end{tabular}

finamiento y del incremento del trabajo a distancia es percibido como positivo por la mayoría de niños y adolescentes (Serrano-Martínez, 2020; Cros et al., 2021). En particular, en un estudio con adolescentes se indica que casi $30 \%$ de los participantes considera que la relación con sus padres, en particular con la madre, mejoró durante el confinamiento (Cros et al., 2021). Sin embargo, es necesario reconocer que la relación con los padres durante este periodo se ve afectada por sus posibilidades para establecer cuidados, afecto y un entorno seguro para los adolescentes (Galiano-Ramírez et al., 2020).

Este resultado es un hallazgo importante considerando que el apoyo emocional, entre otras dimensiones del estilo de crianza de los padres, está relacionado con la conducta prosocial (Mestre, Samper, Tur y Díez, 2001). Por su parte, Meier y Oros (2012) respaldan la idea del impacto significativo de las relaciones paternas en el desarrollo emocional. En particular, Pérez, Romero, Robles y Flórez (2019) identifican el estilo parental autoritativo como favorecedor de conductas prosociales, sobre todo de parte de la madre, quien permanece más tiempo con los jóvenes. Así, es interesante identificar una percep- 
ción en el incremento del apoyo materno durante este periodo de confinamiento en los adolescentes, considerando la posibilidad de que represente un factor protector frente a la generación de diversas problemáticas.

Continuando con las fortalezas externas derivadas de la relación con la madre, en ambos sexos los resultados demostraron una disminución significativa en la percepción de la supervisión materna durante los primeros meses del confinamiento. De manera general, el incremento del teletrabajo o la necesidad de salir a trabajar dejando a los hijos en el hogar, sumado con la educación desde casa, son algunos factores que dificultan que los padres puedan mantener una supervisión estrecha (Nivela-Cornejo et al., 2020). En el periodo de confinamiento se informa que la mayoría de estas tareas se han orientado hacia la madre, favoreciendo una sensación de sobrecarga y una dificultad importante para mantener la supervisión (Galiano-Ramírez et al., 2020).

De acuerdo con Ruvalcaba-Romero, Fuerte-Nava y Robles-Aguirre (2015), el establecimiento de una supervisión que permita la generación de un entorno seguro y estable en la adolescencia representa un factor protector frente a problemáticas psicosociales. En particular, se informa que el control conductual promueve una menor sintomatología depresiva y conductas disruptivas, encontrando una mayor influencia en el control ejercido por la madre. Así, es necesario continuar investigando los factores que permiten a la familia favorecer un ambiente más estable y controlado para el adolescente durante este periodo, buscando generar estrategias para proporcionar ambientes más seguros.

De modo interesante, sólo para las mujeres se encontró una disminución en la percepción de la relación con amigos con hábitos saludables. Diversos estudios hechos durante el confinamiento reportan un incremento de hábitos poco saludables, encontrando un aumento en los problemas de alimentación y sueño, y un decremento de las actividades deportivas (Brooks et al., 2020; Paricio y Pando, 2020; Sandin et al., 2020). Esta situación incrementa la posibilidad de que el adolescente interactúe con pares que también están experimentando una reducción en los hábitos de vida sanos, lo cual, sumado a la incertidumbre de la duración del confinamiento, puede aumentar las posibilidades de desarrollar conductas de riesgo (Paricio y Pando, 2020). Es interesante que esta disminución se haya encontrado sólo en mujeres, siendo importante que futuros estudios analicen si los cambios en hábitos saludables derivados del confinamiento se presentan de manera más significativa en este sexo, así como los posibles factores sociodemográficos que pudieran influir en esta situación.
Respecto a las fortalezas internas, sólo se identificaron diferencias significativas en los hombres, observando una disminución de la responsabilidad y un aumento de la toma de decisiones. De acuerdo con Galiano-Ramírez et al. (2020), de manera natural los adolescentes tienden a tener dificultades para el autocontrol y el seguimiento de reglas, situación que puede ser exacerbada por el confinamiento. En comparación con las mujeres, en el caso de los hombres se informa que tienden a presentar mayor dificultad para autorregular el comportamiento (Álvarez y Moral, 2020). Lo anterior, sumado con la disminución en la supervisión de los padres derivada del confinamiento, podría favorecer una mayor dificultad para cumplir oportunamente con compromisos personales y académicos.

El aumento observado en los hombres en la toma de decisiones durante el confinamiento refleja un incremento en la habilidad para resolver problemas a partir de la recuperación de información, la consideración de posibles consecuencias y distintas formas de solucionar problemáticas. De manera general, diversos estudios indican que los varones tienden a puntuar más alto en distintas dimensiones de la toma de decisiones (Luna-Bernal y Laca-Arocena, 2014). Lo anterior se relaciona quizá con factores sociales derivados de los roles de género, encontrando que las conductas relacionadas con la confianza en las decisiones y la firmeza tienden a ser características esperadas en los hombres (Mejía y Laca, 2006). Por tanto, es posible que las condiciones derivadas de la pandemia y del confinamiento promuevan una mayor promoción de la toma de decisiones consciente en los hombres. Así, es importante continuar el análisis de los factores que afectan los procesos de toma de decisiones en las mujeres, sobre todo durante este periodo de confinamiento que ofrece distintas situaciones de riesgo que el adolescente tiene que afrontar.

Los resultados presentados implican un aporte relevante al permitir conocer las principales diferencias que perciben los adolescentes en sus fortalezas durante los primeros meses del confinamiento. Asimismo, analizar las diferencias que identifican los adolescentes de acuerdo con el sexo permite puntualizar las áreas donde se han fortalecido o afectado las diferentes fortalezas externas e internas. Lo anterior hace posible establecer las fortalezas que se han desarrollado con el confinamiento, así como las que requieren mayor promoción y las que se mantienen aún en los momentos difíciles. La principal limitación de este trabajo incluye el tamaño de la muestra y el proceso de muestreo, siendo necesario desarrollar futuras investigaciones que incluyan mues- 
tras representativas seleccionadas de modo aleatorio para generalizar los resultados.

El confinamiento es una situación que se ha prolongado más de lo esperado y que mantiene una posibilidad importante de postergarse durante un periodo desconocido. Por tanto, es importante que futuros estudios analicen los posibles cambios que se presentan en factores protectores, como las fortalezas durante las diferentes etapas que se han desarrollado del confinamiento. Asimismo, sería relevante analizar las posibles diferencias de acuerdo con el nivel de confinamiento familiar, buscando identificar si es un factor que influye en este tipo de factores protectores. De igual manera, considerando la necesidad de desarrollar intervenciones que promuevan el desarrollo de factores protectores y la prevención de problemáticas psicosociales, es importante explorar los factores individuales, familiares, escolares y sociales que pudieran influir en factores protectores, como las fortalezas.

\section{Conclusiones}

A partir de lo indicado, es posible concluir que los adolescentes perciben cambios en algunas fortalezas externas e internas a partir del confinamiento, y otras se mantienen sin cambios significativos. En particular, para ambos sexos se observaron cambios en las fortalezas derivadas de la relación con la madre, encontrando que durante el confinamiento se percibió un mayor apoyo y una disminución en la supervisión. Se concluye que tal vez esta diferencia se relacione con el incremento en la convivencia con la madre y en las diferentes tareas que se añadieron a la figura materna a partir del confinamiento, sobre todo las derivadas de los cambios en el trabajo, el hogar y la comunidad.

En el caso de las mujeres se observó una disminución en la relación con amigos saludables, concluyendo que quizá se relacione con el incremento generalizado de hábitos sedentarios y alimentación poco saludable reportado en niños, adolescentes y adultos a partir del confinamiento. Lo anterior pudiera favorecer que las interacciones sociales de las mujeres se efectúen con pares que presentan estos mismos hábitos poco sanos, situación que incrementa la posibilidad del desarrollo de conductas de riesgo.

Respecto a los hombres, se encontraron diferencias significativas en las fortalezas internas de responsabilidad y toma de decisiones. De manera general, concluimos que tal vez la falta de supervisión, la dilución de las rutinas y la dificultad para mantener reglas constantes durante el confinamiento pudieran ser factores que favorezcan la disminución de la responsabilidad en los adolescentes varones. Asimismo, es interesante que la toma de decisiones fue una fortaleza donde se indicó un incremento, quizá relacionado con los roles de género donde el tomar decisiones que resuelvan problemas de manera firme y confiada suele ser un atributo más esperado en los hombres.

\section{Referencias}

Álvarez, M., \& Moral, M. (2020). Phubbing, uso problemático de teléfonos móviles y de redes sociales en adolescentes y déficits en autocontrol. Health and Addictions, 20(1), 113125. https://doi.org/10.21134/haaj.v20i1.487

Andrade-Palos, P., Betancourt-Ocampo, D., Moreno-Carmona, N., \& Alvis-Rizzo, A. (2017). Fortalezas externas desde el modelo de desarrollo positivo de los jóvenes y consumo de sustancias en una muestra de adolescentes mexicanos y colombianos. Avances en Psicología Latinoamericana, 35(3), 515-529. http://dx.doi.org/10.12804/revistas. urosario.edu.co/apl/a.4095

Andrade-Palos, P., Betancourt-Ocampo, D., Vallejo-Casarín, A., Segura-Celis, B., \& Rojas-Rivera, M. (2012). Prácticas parentales y sintomatología depresiva en adolescentes. Salud Mental, 35, 29-36. http://www.revistasaludmental. $\mathrm{mx} /$ index.php/salud_mental/article/view/1452/1450

Benson, P. L, Scales, P. C., Hamilton, S. F., \& Sesma Jr., A. (2006). Desarrollo juvenil positivo: Teoría, investigación y aplicaciones. En R. M. Lerner \& W. Damon (eds.), Manual de psicología infantil (6a. ed.). Vol. 1, Modelos teóricos de desarrollo humano, 894-941. Hoboken, Nueva Jersey: John Wiley \& Sons, Inc.

Betancourt-Ocampo, D., Andrade-Palos, P., González, A., \& Sánchez-Xicotencatl, C. (2018). Escala de fortalezas para adolescentes (FIE-A). En Andrade-Palos (coord.), Fortalezas de los adolescentes. México: UNAM.

Betancourt-Ocampo, D., Andrade-Palos, P., \& Marín-Martínez, A. (2018). Análisis psicométrico de la versión revisada de la escala de fortalezas internas y externas para adolescentes (FIE-AR). Revista Colombiana de Ciencias Sociales, 10(1), 20-39. https://doi.org/10.21501/22161201.3058

Brooks, S. K., Webster, R. K., Smith, L. E., Woodland, L., Wessely, S., Greenburg, N., \& Rubin, G. J. (2020). El impacto psicológico de la cuarentena y cómo reducirlo: Revisión rápida de la evidencia. The Lancet, 395, 912-920. https:// doi.org/10.1016/S0140-6736(20)30460-8

Brown, M., Blanchard, T., \& McGrath, R. (2020). Differences in selfreported character strengths across adolescence. Journal of Adolescence, 79, 1-10. https://doi.org/10.1016/j. adolescence.2019.12.008

Cros, B., Preve, P., Revilla, R., \& Maristany, M. (2021). Familia y adolescencia durante la pandemia por COVID-19. Enciclopedia Argentina de Salud Mental. Recuperado de ENCICLOPEDIA ARGENTINA DE SALUD MENTAL (enciclopediasaludmental.org.ar)

Florenzano, R., Valdés, M., Cáceres, C., Casassus, M., Sandoval, A., Santander, S., \& Calderón, S. (2009). Percepción de la relación parental entre adolescentes mayores y menores 
de 15 años. Revista Chilena de Pediatría, 80(6), 520-527. https://doi.org/10.4067/S0370-41062009000600004

Galiano-Ramírez, M. C., Prado-Rodríguez, R., \& MustelierBecquer, R. (2020). Salud mental en la infancia y adolescencia durante la pandemia de COVID-19. Revista Cubana de Pediatría, 92. http://www.revpediatria.sld.cu/ index.php/ped/article/view/1342/681

García-Álvarez, D., Hernández-Lalinde, J., Cobo-Rendón, R., Espinosa-Castro, J., \& Soler, M. (2020). Medición de las fortalezas del carácter en adolescentes y su predicción en el afrontamiento y bienestar psicológico. Archivos Venezolanos de Farmacología y Terapéutica, 39(4), 303317. http://saber.ucv.ve/ojs/index.php/rev_aavft/article/ view/20715/144814487027

Gómez-León, M. (2021). Disminución de la ansiedad en las víctimas del bullying durante el confinamiento por COVID-19. Revista de Educación a Distancia, 65(21), https://doi.org/10.6018/red.439601

González-Valero, G., Zurita-Ortega, F., Lindell-Postigo, D., Conde-Pipó, J., Grosz, W. R., \& Badicu, G. (2020). Analysis of Self-Concept in Adolescents before and during COVID-19 Lockdown: Differences by gender and sports activity. Sustainability, 12(18). https://doi.org/10.3390/ su12187792

Janssen, L. H., Kullberg, M. L. J., Verkuil, B., Van Zwieten, N., Wever, M. C., Van Houtum, L. A., Wentholt, W. G., \& Elzinga, B. M. (2020). Does the COVID-19 pandemic impact parents' and adolescents' well-being? An EMA-study on daily affect and parenting. PloS one, 15(10), e0240962. https://doi. org/10.1371/journal.pone.0240962

Lerner, R., \& Lerner, J. (2011). The Positive Development of Youth. Report of the Findings from the First Seven years of the 4-H Study of Positive Youth Development. Institute for Applied Research in Youth Development Tufts University.

Luna-Bernal, A. C. A., \& Laca-Arocena, F. A. V. (2014). Patrones de toma de decisiones y autoconfianza en adolescentes bachilleres. Revista De Psicología, 32(1), 39-65. https:// doi.org/10.18800/psico.201401.002

Martínez-Pérez, A., Fernández-Fernández, V., Alcántara-López, M., López-Soler, C., \& Castro-Sáez, M. (2020). Resultados preliminares del impacto de la COVID-19 en niños/niñas y adolescentes expuestos a maltrato intrafamiliar. Terapia Psicológica 38(3), 427-445. https://doi.org/10.4067/ S0718-48082020000300427

Meier, L. K., \& Oros, L. B. (2012). Percepción de las prácticas parentales y experiencia de emociones positivas en adolescentes. Revista de Psicología, 9(16), 73-84.

Mejía, J. C., \& Laca, F. A. (2006). Estilos de comunicación en el conflicto y confianza en las propias decisiones. Enseñanza e Investigación en Psicología, 11(2), 347-358.

Mestre, M. V., Samper, P., Tur, A., \& Díez, I. (2001). Estilos de crianza y desarrollo prosocial de los hijos. Revista de Psicología General y Aplicada, 54(4), 691-703. http:// www.codajic.org/sites/www.codajic.org/files/Dialnet-Estil osDeCrianzaYDesarrolloProsocialDeLosHijos-2364995 O. pdf

Moreno-Carmona, N. D., Andrade-Palos, P., \& BetancourtOcampo, D. (2018). Fortalezas en adolescentes de Colombia y México que nunca han intentado suicidarse. Revista Latinoamericana de Ciencias Sociales, Niñez y Juventud, 16(2), 797-807. https://doi. org/10.11600/1692715x.16210

Nivela-Cornejo, M., Molina-Villacís, C., \& Campos-Vera, R. (2020). El rol de la familia en la educación en casa durante el confinamiento. Journal of Business Science, 2(6), 22-29. https://revista.estudioidea.org/ojs/index.php/eidea/ article/view/38

Ovejero, M., Cardenal, V., \& Ortiz-Tallo, M. (2016). Fortalezas humanas y bienestar biopsicosocial: Revisión sistemática. Escritos de Psicología, 9(9), 4-14. http://doi.org/10.5231/ psy.writ.2016.2311

Palacio-Ortiz, J. D., Londoño-Herrera, J. P., Nanclares-Márquez, A., Robledo-Rengifo, P., \& Quintero-Cadavid, C. (2020). Trastornos psiquiátricos en los niños y adolescentes en tiempo de la pandemia por COVID-19. Revista Colombiana de Psiquiatría, 49(4) 279-288. https://doi.org/10.1016/j. rcp.2020.05.006

Paricio, R., \& Pando, M. (2020). Salud mental infanto-juvenil y pandemia de Covid-19 en España: Cuestiones y retos. Revista de Psiquiatría Infanto-Juvenil, 37(2), 30-44. https://doi.org/10.31766/revpsij.v37n2a4

Pérez, K. L., Romero, K., Robles, J. L., \& Flórez, M. (2019). Prácticas parentales y su relación con conductas prosociales y agresivas en niños, niñas y adolescentes de instituciones educativas. Revista Espacios, 40(31). https://www. revistaespacios.com/a19v40n31/a19v40n31p08.pdf

Reyes, S., \& Ferragut, M. (2016) Fortalezas psicológicas y diferencias de sexo en adolescentes. Escritos de Psicología, 9(3), 28-36. http://dx.doi.org/10.5231/psy. writ.2016.2811

Ruiz-Roso, M. B., De Carvalho Padilha, P., Mantilla-Escalante, D. C., Ulloa, N., Brun, P., Acevedo-Correa, D., Ferreira Peres, W. A., Martonell, M., Tschoepke, M., De Oliveira, L., Carrasco-Marin, F., Paternina-Sierra, K., Rodrigez-Meza, J., Montero, P., Bernabé, G., Pauletto, A., Taci, X., Visioli, F., \& Dávalos, A. (2020). Covid-19 confinement and changes of adolescent's dietary trends in Italy, Spain, Chile, Colombia and Brazil. Nutrients, 12(6), 1807. https://doi. org/10.3390/nu12061807

Ruvalcaba-Romero, N., Fuerte-Nava, J. M., \& Robles-Aguirre, F. (2015). Comunicación con padres y docentes como factor protector de los adolescentes ante las conductas disociales. Educación y Ciencia, 4(44), 57-67.

Ruvalcaba-Romero, N., Gallegos-Guajardo, J., Caballo, M. V. E., \& Villegas-Guinea, D. (2016). Prácticas parentales e indicadores de salud mental en adolescentes. Psicología desde el Caribe, 33(3), 223-236.

Ruvalcaba-Romero, N., Orozco-Solís, M., Gallegos-Guajardo, J., \& Nava-Fuerte, J. (2018). Relaciones escolares, comunicación con padres y prosocialidad como predictores de emociones positivas. Liberabit, 24(2), 183-193. https:// doi.org/10.24265/liberabit.2018.v24n2.02

Salin, M., Kaittila, A., Hakovirta, M., \& Anttila, M. (2020). Family coping strategies during Finland's COVID-19 lockdown. Sustainability, 12(21), 9133. https://doi.org/10.3390/ su12219133

Sánchez-Xicoténcatl, C. O., \& Andrade-Palos, P. A. (2019). Fortalezas en adolescentes que no consumen 
tabaco. Psicología Iberoamericana, 27(2). https:// psicologiaiberoamericana.ibero.mx/index.php/psicologia/ article/view/47/162

Sandín, B., Valiente, R., García, J., \& Chorot, P. (2020). Impacto psicológico de la pandemia de COVID-19: Efectos negativos y positivos en población española asociados al periodo de confinamiento nacional. Revista de Psicopatología y Psicología Clínica, 21(1), 1-22. http://doi.org/10.5944/ rppc.27569

Sekulic, D., Blazevic, M., Gilic, B., Kvesic, I., \& Zenic, N. (2020). Prospective analysis of levels and correlates of physical activity during COVID-19 pandemic and imposed rules of social distancing. Gender specific study among adolescents from Southern Croatia. Sustainability, 12(10), 4072. https://doi.org/10.3390/su12104072

Serrano-Martínez, C. (2020). Impacto emocional y crianza de menores de cuatro años durante el COVID-19. Perifèria, Revista de Recerca i Formació en Antropologia, 25(2), 7487. https://doi.org/10.5565/rev/periferia.735

Van de Groep, S., Zanolie, K., Green, K. H., Sweijen, S. W., \& Crone, E. A. (2020). A daily diary study on adolescents' mood, empathy, and prosocial behavior during the COVID-19 pandemic. PloS one, 15(10), e0240349. https:// doi.org/10.1371/journal.pone.0240349

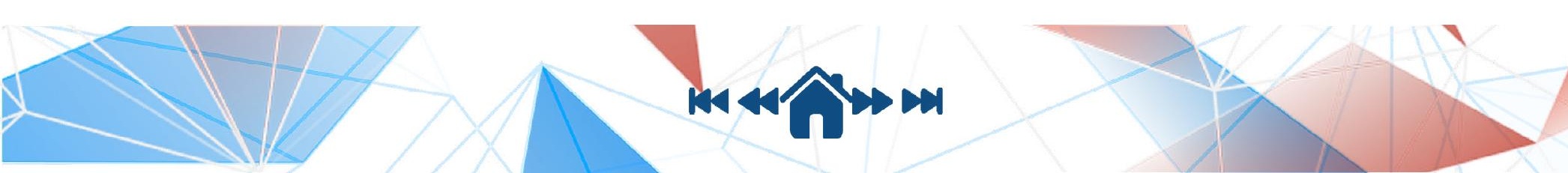




\section{Meta-Análisis del Artículo}




\section{Dimensión Cuantitativa}

\section{Perfil de Evaluación entre pares}
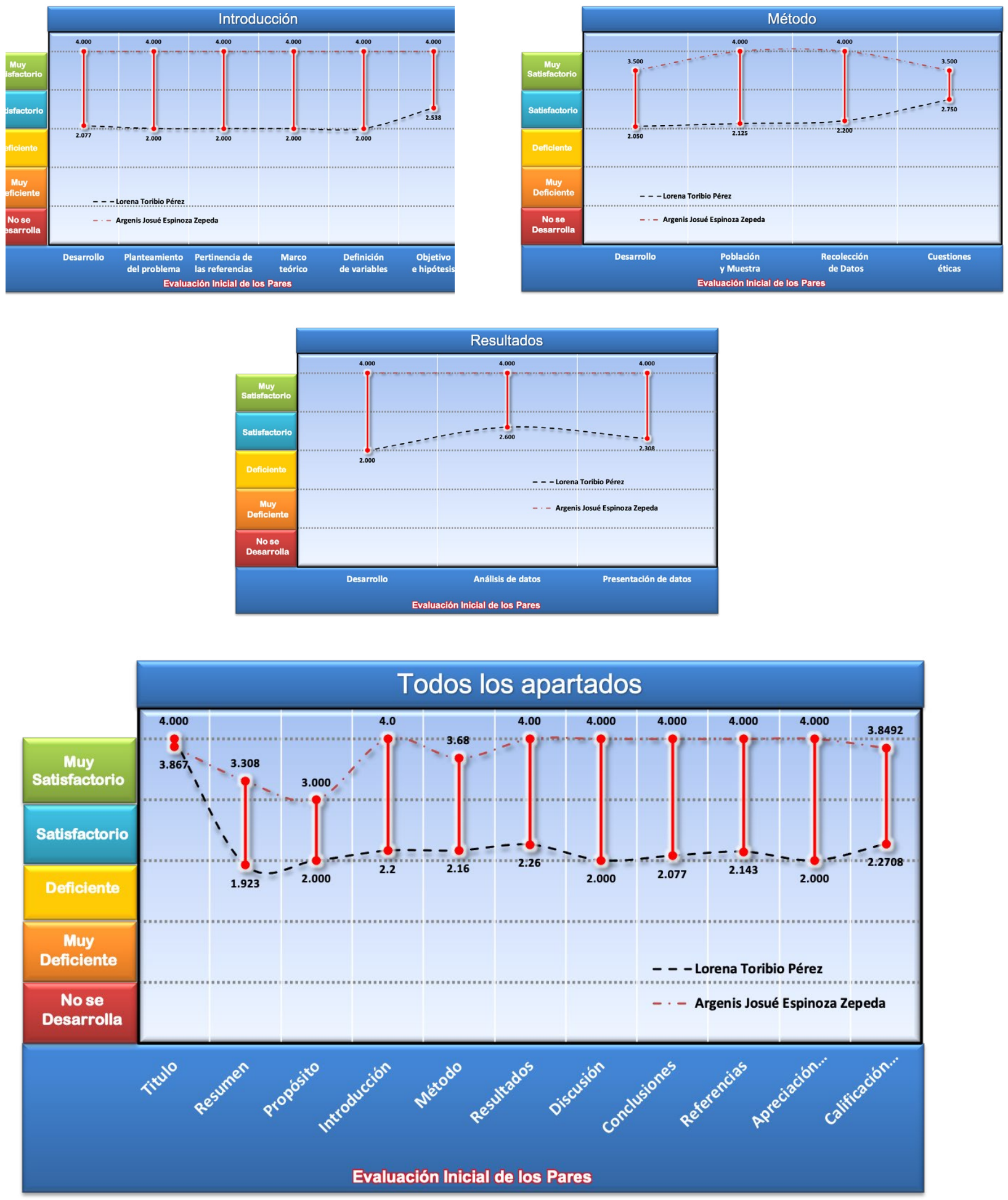


\section{Índice de Concordancia}

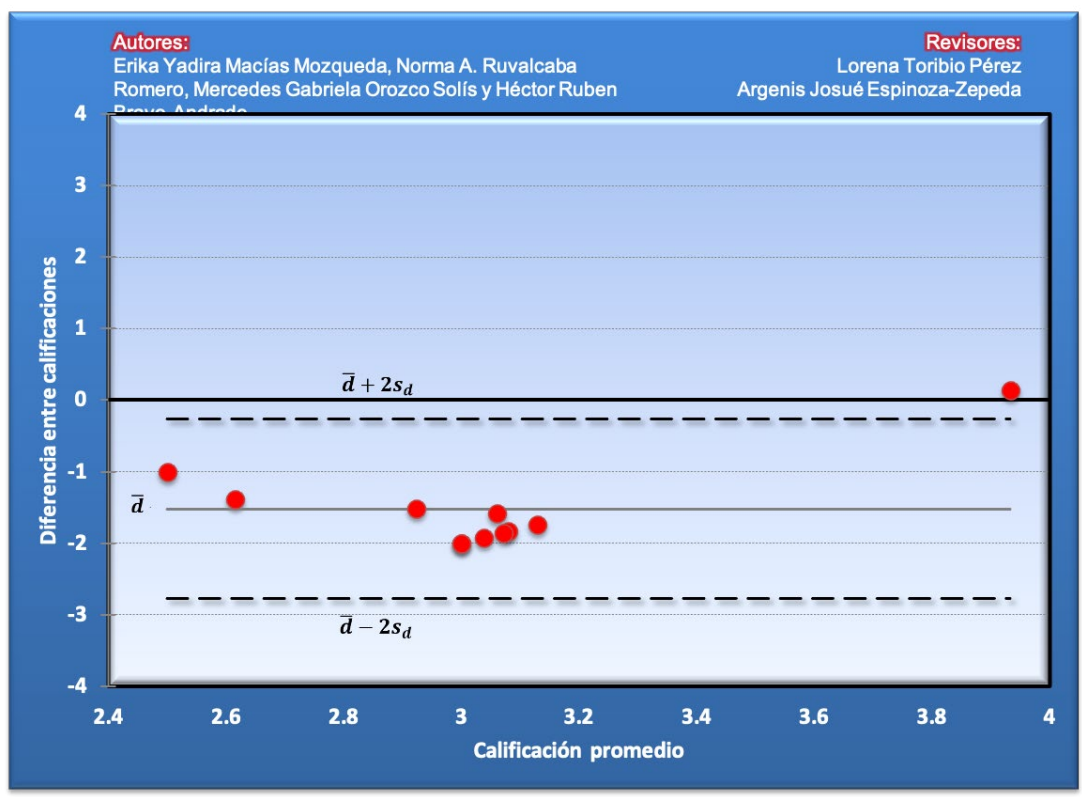

\section{Índice de Acuerdo}

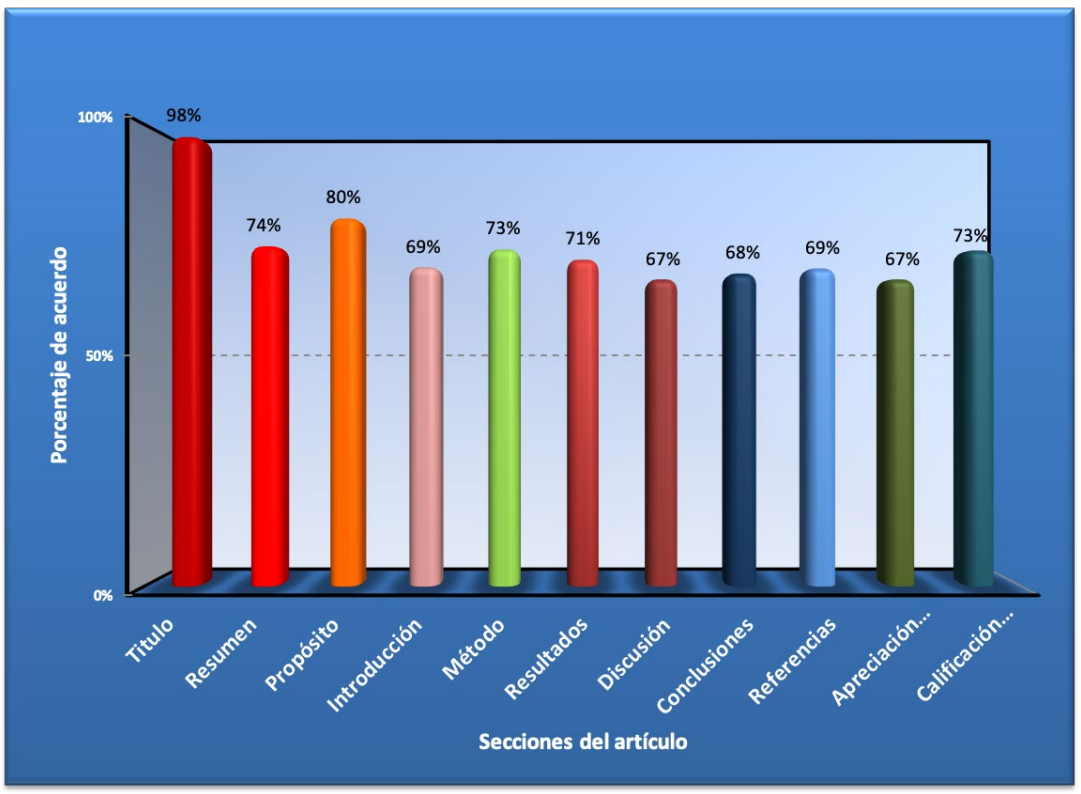


Dimensión Cualitativa

\begin{tabular}{|c|c|}
\hline Revisor 1 & Revisor 2 \\
\hline Lorena Toribio Pérez & Argenis Josué Espinoza Zepeda \\
\hline \multicolumn{2}{|c|}{ Título/Autoría } \\
\hline El título del manuscrito es correcto & No hay comentarios \\
\hline \multicolumn{2}{|c|}{ Resumen } \\
\hline $\begin{array}{l}\text { Incluir en el resumen resultados con valores numéricos } \\
\text { según corresponda. Incluir en el resumen las caracte- } \\
\text { rísticas de la muestra: total de la muestra, edad y sexo }\end{array}$ & $\begin{array}{l}\text { El resumen no reporta resultados con valores numéricos. } \\
\text { Tiene } 152 \text { palabras. }\end{array}$ \\
\hline \multicolumn{2}{|c|}{ Próposito del Estudio } \\
\hline Incluir la hipótesis de la investigación & No hay comentarios \\
\hline \multicolumn{2}{|c|}{ Introducción } \\
\hline Incluir las hipótesis de la investigación & No hay comentarios. \\
\hline \multicolumn{2}{|c|}{ Método } \\
\hline $\begin{array}{l}\text { Describir la validez y confiablidad de los instrumentos } \\
\text { utilizados. Describir si se obtuvo autorización del comité } \\
\text { de ética de la institución que llevó a cabo la investiga- } \\
\text { ción. Mencionar las consideraciones éticas que se infor- } \\
\text { maron a los participantes. }\end{array}$ & No hay comentarios \\
\hline \multicolumn{2}{|c|}{ Resultados } \\
\hline $\begin{array}{l}\text { Se recomienda incluir una tercer tabla con los resultados } \\
\text { de la muestra general. El objetivo del estudio fue identifi- } \\
\text { car la percepción que tienen los adolescentes de sus for- } \\
\text { talezas internas y externas antes y durante los primeros } \\
\text { meses del confinamiento. Por lo que es importante incluir } \\
\text { los datos de las diferencias que existen antes y durante el } \\
\text { confinamiento en la muestra general. }\end{array}$ & No hay comentarios \\
\hline \multicolumn{2}{|c|}{ Discusión } \\
\hline $\begin{array}{l}\text { Agregar en la discusión el análisis de los resultados de } \\
\text { la muestra general de las fortalezas antes y durante el } \\
\text { confinamiento. }\end{array}$ & No hay comentarios \\
\hline
\end{tabular}


Artículo Empírico | Percepción de las fortalezas de los adolescentes...| Macías-Mozqueda, Ruvalcaba-Romero, Orozco-Solís, y Bravo-Andrade

\section{Revisor 1}

Revisor 2

\section{Conclusiones}

Concluir en relación a las características de las fortalezas antes y durante el confinamiento en la muestra general.

No hay comentarios

\section{Referencias}

Revisar las citas, en algunas se mencionan a mas de 5 autores y en otras de menos de 5 autores se coloca et al en la primera cita. Se mencionan por segunda vez todos los autores, cuando lo correcto es que en la segunda cita se coloca et al., No se recomienda citar el mismo autor en un mismo párrafo Revisar con atención citas y referencias para que coincidan.

No hay comentarios 
